\title{
Reducing urban traffic congestion due to localized routing decisions
}

\author{
Bo Li $\odot,{ }^{1, *}$ David Saad $\odot,{ }^{1, \dagger}$ and Andrey Y. Lokhov ${ }^{2, *}$ \\ ${ }^{1}$ Non-linearity and Complexity Research Group, Aston University, Birmingham B4 7ET, United Kingdom \\ ${ }^{2}$ Theoretical Division, Los Alamos National Laboratory, Los Alamos, New Mexico 87545, USA
}

(Received 25 February 2020; revised 8 July 2020; accepted 20 August 2020; published 2 September 2020)

\begin{abstract}
Balancing traffic flow by influencing drivers' route choices to alleviate congestion is becoming increasingly more appealing in urban traffic planning. Here, we introduce a discrete dynamical model comprising users who make their own routing choices on the basis of local information and those who consider routing advice based on localized inducement. We identify the formation of traffic patterns, develop a scalable optimization method for identifying control values used for user guidance, and test the effectiveness of these measures on synthetic and real-world road networks.
\end{abstract}

DOI: 10.1103/PhysRevResearch.2.032059

Many of the world's major cities are increasingly gridlocked with a staggering estimated annual cost of $\$ 166 \mathrm{~B}$ in the United States alone [1]. Relentless urban population growth has created exorbitant traffic demands, which leads to recurring large-scale traffic jams [2-4]. Since it is expensive to satisfy the demand exclusively through further investment in infrastructure, there is a growing interest in optimizing transportation systems within the existing infrastructure [5-7]. Modern information technologies can potentially offer effective solutions through ride sharing using smart phones [8], congestion-aware routing schemes [9], and the use of autonomous vehicles $[10,11]$. The deployment of smart devices already impacts transportation networks, leading to a paradigm shift in traffic planning and management. However, not all these changes are for the better. Most navigation apps have been designed typically to minimize an individual driver's travel time irrespective of street capacity along the route, safety, or the route choices of other drivers; in many times this results in traffic chaos [12]. Moreover, recent simulation results demonstrate the potential of having a mixed environment, of drivers who make their own route choices en route and those who follow routing advice that is centrally optimized, in reducing congestion [13]; this scenario is inherently accommodated within the framework presented here. It is therefore important to understand the potential and limitations of these technologies and develop scalable algorithmic tools that would enable their use in real-time settings.

Detailed microscopic modeling of multiagent systems characterizing the paths of individual users, such as cellular automata-based simulations [14], model basic traffic systems

\footnotetext{
*b.li10@aston.ac.uk

†.saad@aston.ac.uk

†lokhov@lanl.gov
}

Published by the American Physical Society under the terms of the Creative Commons Attribution 4.0 International license. Further distribution of this work must maintain attribution to the author(s) and the published article's title, journal citation, and DOI. but usually require considerable computational power; it is also generally difficult to gain insight due to the overwhelming level of details. On the other hand, models based on traffic flow, that coarse-grain the behaviors of individual users but maintain correlations at the network level, are simplistic but amenable to analysis. Link-based methods have been developed along this line, mainly for static assignments, selfish routing, or centralized optimization $[15,16]$. Such methods have also been extended to the more difficult dynamic traffic assignment problem [17]; for instance, the Wardrop's static equilibrium principle was extended to dynamic scenarios [18]. In reality, drivers do not have full information of the traffic flow and unbounded computational capacity to determine the rational route choices $[19,20]$. Instead, they typically adjust their route choice, especially in urban settings, en route according to the traffic conditions in downstream junctions, which has been investigated in some dynamic traffic assignment problems [21-23].

In this Rapid Communication, we take into account such behavioral aspects and propose a dynamical model which includes both impulsive users who make their own decisions en route, and advice-susceptible users who follow the suggestions given by smart devices. Advice-susceptible users are incentivized to follow centrally optimized routing suggestions that benefit traffic globally. Such a strategy may be adopted in the future to alleviate traffic congestion [13]. In fact, electronic road pricing already operates successfully in Singapore [24], and has been recently launched in Israel to motivate drivers into driving in nonrush hours and carpooling [25]. Our computational model offers complementary insights in support of such strategies. We focus on scenarios where commuters travel towards the city center at peak hours, during which they typically experience severe traffic congestion. A realistic model of this type is naturally nonlinear, and hard to optimize; one of the contributions of this Rapid Communication consists in developing a scalable and computationally efficient optimization method that supports real-time applications. We analyze the characteristics of emerging traffic patterns, develop an algorithm to determine the optimal incentive, and investigate their impact on traffic congestion. 
We model the urban road system as a network, where intersections are mapped to nodes and roads between them to edges (or links). We consider a scenario where drivers travel towards a universal destination $D$, which is relevant in the morning rush hour when a large number of people commute to the city center. The network is depicted as an undirected graph $G(V, E)$ of $N$ nodes, where each node $i \in V$ is connected to $k_{i}$ neighbors denoted by $\partial i$, and each edge $(i, j) \in E$ represents two lanes $i \rightarrow j$ and $j \rightarrow i$, accommodating noninteracting traffic from $i$ to $j$ and $j$ to $i$, respectively. We denote the set of all lanes as $\mathcal{E}$.

Assume that drivers can be classified into two groups according to whether they make their own route choices or follow the advice from navigation devices. In the former, a user makes routing decisions dynamically, based on her estimated time to destination $D$. Upon arriving at intersection $i$ at time $t$, the user faces a choice between $k_{i}$ possible roads $\{i \rightarrow j\}_{j=1}^{k_{i}}$. The user first estimates (i) the time it takes to travel through edge $i \rightarrow j$ as $g\left(\rho_{i j}^{t}\right)$ where $\rho_{i j}^{t}$ is the number of users occupying edge $i \rightarrow j$ (i.e., traffic volume) at that time and $g\left(\rho_{i j}^{t}\right)$ is determined by the Greenshields model [26] [see also the Supplemental Material (SM) [27]], and (ii) the remaining time $d_{j}$ needed to travel to $D$ from node $j$, which can be taken as the shortest free traveling time or be based on past experience of the congestion level. Afterwards, their route choices are made according to the probability

$$
p_{i j}^{g, t}\left(\rho^{t}\right)=\frac{e^{-\beta\left[g\left(\rho_{i j}^{t}\right)+d_{j}\right]}}{\sum_{k \in \partial i} e^{-\beta\left[g\left(\rho_{i k}^{t}\right)+d_{k}\right]}},
$$

where $\beta$ is a parameter determining the randomness of the decision-making process. As shown in the SM [27], the dependence on $\beta \geqslant 1$ is relatively weak, and hence we choose $\beta=1$ in what follows. Note that we do not limit users from turning back. The awareness of congestion can be extended to road segments that are more distant, at the cost of higher computational complexity. Here, we focus on the one-step congestion-aware model.

In the latter group, users follow the navigation advice aimed at improving traffic efficiency. Their route choices at junction $i$ at time $t$ are determined by the localized probability

$$
p_{i j}^{w, t}\left(w^{t}\right)=e^{-w_{i j}^{t}} / \sum_{k \in \partial i} e^{-w_{i k}^{t}},
$$

where the weight variables $\left\{w_{i j}^{t}\right\}$ are optimized centrally. With the assumption that the fraction of users $n$ who are susceptible to routing advice are distributed evenly in the network, on average the vehicle flow arriving at node $i$ at time $t$ will be diverted to the adjacent edges $\{i \rightarrow j\}_{j=1}^{k_{i}}$ according to the distribution

$$
p_{i j}^{t}\left(\rho^{t}, w^{t}\right)=(1-n) p_{i j}^{g, t}\left(\rho^{t}\right)+n p_{i j}^{w, t}\left(w^{t}\right) .
$$

A similar decision rule has been used to investigate the effect of altruistic users in the static routing game setting [7,28], which differs from the current dynamical model.

At each time step $t^{\prime}$ a decision is made to enter edge $i \rightarrow j$, the user then spends some time $\tau_{i j}$ traveling on this edge with distribution $P\left(\tau_{i j}\right)$, arriving at the end point at time $t=t^{\prime}+\tau_{i j}$. The distribution of time spent can take several forms, including the typically used discrete Poisson distribution adopted here [27]. The arrival probability depends on the traffic volume $\rho_{i j}^{t^{\prime}}$ at the time of entrance $t^{\prime}$, i.e., $P\left(t-t^{\prime} \mid \rho_{i j}^{t^{\prime}}\right)$, which is a realistic and an important factor in traffic modeling. To express the dynamics we introduce the time-dependent flux $f_{i j}^{t}$ arriving at the end point $j$ of the edge $i \rightarrow j$ at time $t$. Assuming users enter the road system at time $t=0$ with initial volume $\rho^{0}$, the dynamics of the traffic volume and flux on edge $i \rightarrow j(i \neq D)$ are governed by the discrete forward dynamics

$$
\begin{gathered}
\rho_{i j}^{t}=p_{i j}^{t-1} \sum_{k \in \partial i, k \neq D} f_{k i}^{t-1}+\left(\rho_{i j}^{t-1}-f_{i j}^{t-1}\right), \\
f_{i j}^{t}=\sum_{t^{\prime}=1}^{t}\left[\rho_{i j}^{t^{\prime}}-\left(\rho_{i j}^{t^{\prime}-1}-f_{i j}^{t^{\prime}-1}\right)\right] P\left(t-t^{\prime} \mid \rho_{i j}^{t^{\prime}}\right)+\rho_{i j}^{0} P\left(t \mid \rho_{i j}^{0}\right) .
\end{gathered}
$$

Equation (4) describes the traffic volume at edge $i \rightarrow j$ at time $t$; it is composed of the newly joined users who selected this junction at node $i$ at time $t-1$, and users who were already traveling through this edge but have not yet reached the end point $j$. Equation (5) states that the vehicles flux at the edge $i \rightarrow j$ end point at time $t$ comprises the fraction of traffic volume $\rho_{i j}^{t^{\prime}}-\left(\rho_{i j}^{t^{\prime}-1}-f_{i j}^{t^{\prime}-1}\right)$ entering the road segment at $t^{\prime}$, who have completed the trip on this road segment within a duration $t-t^{\prime}$ as dictated by the probability $P\left(t-t^{\prime} \mid \rho_{i j}^{t^{\prime}}\right)$, which is defined such that the mean traveling time follows the Greenshields model [26,27]. The resulting model bears similarity to certain link-based models of dynamic traffic assignment [17]. We assume that no vehicles leave the destination node, i.e., the destination $D$ is an absorbing boundary which satisfies $\rho_{D j}^{t}=f_{D j}^{t}=0, \forall j \in \partial D$.

The model is simulated for a fixed time window $T$. To evaluate the efficiency of the system, we measure the average time to destination $D$ ahead of $T$,

$$
\mathcal{O}=\frac{1}{\sum_{e \in \mathcal{E}} \rho_{e}^{0}} \sum_{t=1}^{T}(T-t) \sum_{j \in \partial D} f_{j D}^{t},
$$

and use it as the main performance measure. Other measures can be easily accommodated within the same framework but will not be considered here.

We perform numerical experiments on both generated and realistic road networks. The former are constructed by randomly rewiring a planar square lattice with shortcut edges, which is motivated by the recent observation that high-speed urban roads constitute effective long-range connections and render the system to exhibit small-world characteristics [29]. The realistic road network used is extracted from the OpenStreetMap data set [30], and converted to a network format by using the GIS F2E software [31]. Two examples of the networks considered are shown in Fig. 1. Details of the network generation are described in the SM [27].

Model characterization without control. The initial traffic volume is assigned independently and identically at random as $\rho_{i}^{0}$ users departing from each node $i$, which can be proportional to the population at that node; users rest on the node's neighboring edges $\{i \rightarrow j \mid j \in \partial i\}$ with equal probability $\rho_{i j}^{0}=\rho_{i}^{0} / k_{i}$, constituting the initial traffic volume $\left\{\rho_{i j}^{0}\right\}$. 
(a)

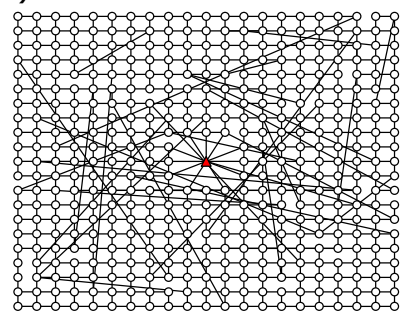

(b)

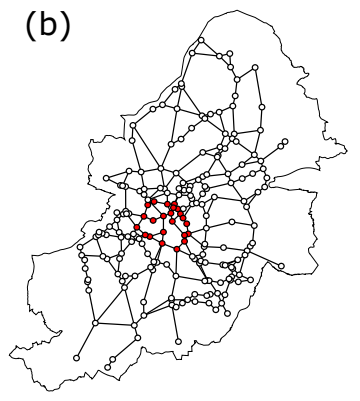

FIG. 1. (a) A small-world network generated by rewiring a $21 \times$ 21 square lattice with shortcut links with rewiring probability $p_{r}=$ 0.05. (b) The Birmingham road network composed of major roads in the city of Birmingham, U.K. We define the city center as the the region enclosed by ring road $\mathrm{A} 4540$. In both cases, the red nodes constitute the city center and determine the destination in the model.

After entering the system at time $t=0$, all users drive towards the center node $D$ according to the instantaneous decisionmaking rule of Eq. (1), i.e., $n=0$ in Eq. (3). Clearly, the same framework can accommodate users entering the network at any time. It leads to macroscopic dynamical traffic patterns governed by Eqs. (4) and (5). We define the traffic load level as

$$
L=\frac{\sum_{e \in \mathcal{E}} \rho_{e}^{0}}{\sum_{e \in \mathcal{E}} \rho_{e}^{\mathrm{jam}}} .
$$

The load level $L$ is similar to the demand-to-supply ratio introduced in Ref. [7], which is suggested to be a good predictor of the congestion level.

We first study emerging traffic patterns in the absence of routing advice, $n=0$. The movement of traffic mass can be visualized by contrasting the traffic volume $\rho_{e}$ to the distance to destination of each lane. To this end, we define the distance $\operatorname{dist}(e, D)$ of lane $e=(i \rightarrow j)$ to destination $D$ as the shortest free traveling time from the midpoint of the lane to destination $\operatorname{dist}(e, D)=d_{j}+t_{i j}^{\mathrm{free}} / 2$. Figure 2 demonstrates how the average traffic volumes $\left\langle\rho_{e}^{t}\right\rangle$ at specific distances change over time under two different load levels. At the low load regime $L=0.1$, shown in Fig. 2(a), the vehicles are able to move fairly quickly towards the destination $D$ from the initial positions at $t=0$ to $t=25$. The roads near the city center become congested, leading to a slow clearance of traffic from $t=50$
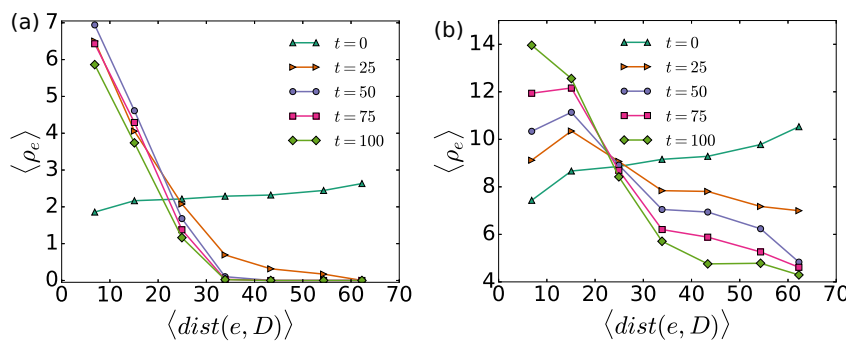

FIG. 2. Average volume $\left\langle\rho_{e}^{t}\right\rangle$ vs average distance to destination $\operatorname{dist}(e, D)$ in a $21 \times 21$ small-world network. Maximal time is $T=$ 100 and users are unaided $n=0$. The road sections are first binned into groups according to dist $(e, D)$ in the interval of 10 , after which $\rho_{e}^{t}$ and $\operatorname{dist}(e, D)$ are averaged within each group. (a) Load level $L=$ 0.1 . (b) Load level $L=0.4$.
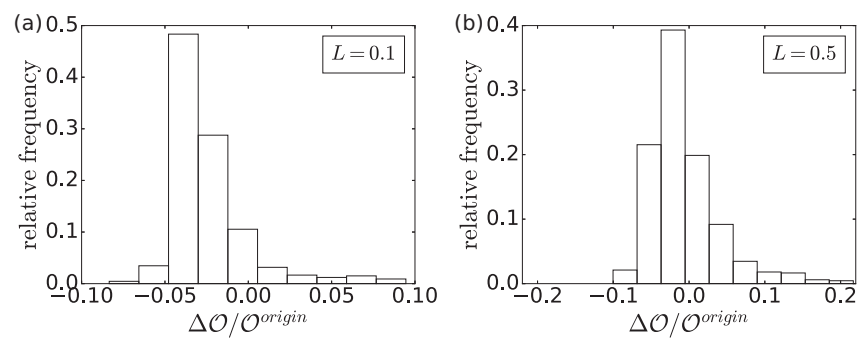

FIG. 3. Relative frequency of fractional change of the objective function $\mathcal{O}$ after adding a link to the existing network, defined as the performance change $\Delta \mathcal{O}=\mathcal{O}^{\text {add-link }}-\mathcal{O}^{\text {origin }}$ divided by the objective function before adding a link $\mathcal{O}^{\text {origin }}$. The results are aggregated from ten networks generated from the small-world network model of size $21 \times 21$ with rewiring probability $p_{r}=0.05$. The parameters are $T=100, n=0$. One end of the new link is randomly chosen from the top five sites with the highest population, while the other end is randomly chosen from the nearest neighbors of the destination node. (a) Load level $L=0.1$. (b) Load level $L=0.5$.

to $t=100$, which indicates that the limited connectivity of the city center is a bottleneck of the traffic system. At high loads $L=0.4$, shown in Fig. 2(b), the traffic volumes at large distance to destination decrease, while those at short distances increase over time, but at a much slower rate compared to the case of a small load $L=0.1$. It indicates that the excessive demand creates congestion in the transportation network and leads to an increase in travel time. More details of the system efficiency as a function of load are depicted in the SM [27].

The simplest measure to reduce congestion is to improve the infrastructure, e.g., by building new roads or by increasing the capacity of existing ones. In particular, increasing the number of possible routes to the city center/destination node can significantly enhance the traffic clearance rate, yet it is rarely possible to do so due to the limited land availability. To examine the effect of network extension, we perform experiments by adding links from sites with the largest populations to nearest neighbors of the destination node. From the relative frequency of the fractional change of objective function shown in Fig. 3, it is surprising to observe that the majority of link additions lead to a decrease in system performance. It suggests that newly introduced shortcuts, being attractive to users, create congestion in the shortcut edges and nearby areas. The phenomenon is reminiscent of Braess's paradox in the static routing game [32] and other complex systems [33-35], where adding resources can possibly lead to a degradation of system performance. In our model, drivers have limited knowledge and are unaware of the long-distance traffic condition, so that the myopic decisions make the system more prone to congestion. If users are aware of more global information as in routing game scenarios, it is possible that they may adapt, in a repeated game scenario, to avoid the already congested shortcuts, such that the probability of performance decrease becomes smaller.

Nevertheless, there is a small likelihood that adding a new link would lead to a significant improvement of the objective function $\mathcal{O}$, which can be up to $10 \%$ for $L=0.1$ and $20 \%$ for $L=0.5$. Such an improvement is more commonly observed in higher loads, but in the majority of cases the improvement is marginal. In either case, it is crucial to select the correct 

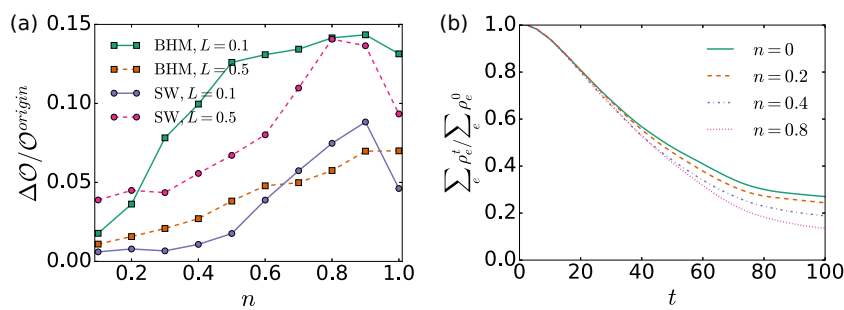

FIG. 4. (a) Fractional change in objective function $\mathcal{O}$ (defined as the performance change $\Delta \mathcal{O}=\mathcal{O}^{\text {optimized }}-\mathcal{O}^{\text {origin }}$ divided by the objective function without advice-susceptible users $\mathcal{O}^{\text {origin }}$ ) as a function of the fraction of advice-susceptible users $n$. The Birmingham road network $(\mathrm{BHM})$ and a small-world network (SW) of size $21 \times 21$ are considered. (b) Time evolution of the fraction of traffic volume $\sum_{e} \rho_{e}^{t} / \sum_{e} \rho_{e}^{0}$ remaining on the Birmingham road network; at time $t=0$, the system load is $L=0.1$.

shortcut to invest in, which becomes a difficult task when the demand profile is fluctuating.

Model characterization with control. It becomes increasingly more appealing and cost effective to influence the route choice of drivers in order to reduce congestion. We examine the particular type of instantaneous advice in the form of Eq. (2), which is adapted such that the objective function $\mathcal{O}$ of Eq. (6) is maximized. The resulting highly nonlinear optimization problem is nonconvex and suffers from multiple local maxima.

To solve this difficult optimization problem, we adopt an optimal control framework [36,37], whereby the dynamics, Eqs. (4) and (5), is enforced as constraints in the Lagrangian formulation. The optimality conditions lead to a set of coupled nonlinear equations solved by forward-backward iterations. To suppress divergent behavior due to radical changes of the control parameters [36,38], we employ a gradient ascent in the updates of the control parameters [27]. Our method achieves similar objective function values to stateof-the-art constrained optimization approaches, while offering significant stability and scalability advantages. This point is illustrated in the SM through a benchmarking comparison to the state-of-the nonlinear programming solver IPOPT [27]. The results shown in Fig. 4 demonstrate that the optimization algorithm successfully improves the system performance, as indicated by the fractional increase in the objective function $\mathcal{O}$ compared to the value $\mathcal{O}^{\text {origin }}$ without advice-susceptible users. The maximal average improvements are remarkably significant and range from $7 \%$ to $14 \%$, depending on the network structure and load level. Naively, one would expect for the objective function to monotonically increase with $n$. However, it seems not to be the case in the experiment shown in Fig. 4(a) and a slight decrease in performance is shown close to $n=1$. For $n<1$, the mixture probability $p_{i j}^{t}=(1-n) p_{i j}^{g, t}+n p_{i j}^{w, t}\left(w^{t}\right)$ includes information on the unguided users and effective distance to destination through $p_{i j}^{g, t}$, which facilitates the search for an optimal solution. This information is gradually lost at high $n$ values, resulting in a less pronounced increased performance, compared to the maximally achieved level of gain.

In Fig. 4(b), we demonstrate the evolution of the fraction of traffic $\sum_{e} \rho_{e}^{t} / \sum_{e} \rho_{e}^{0}$ remaining on the Birmingham road network at a given time as a function of the guided-users fraction $n$. One can observe a faster rate of traffic decrease when $n$ increases from 0 to 0.8 , suggesting more users can reach the destination within the same time period with the increase in the number of advice-susceptible users.

Modeling the dynamics of a transportation network that accommodates different driver behaviors facilitates a greater understanding of the emerging traffic patterns in a regime that is of great interest and relevance [13], while the suggested optimization scheme provides a scalable and efficient way to implement it, providing better performance than state-of-theart continuous optimization solvers and offering significant advantages in the online setting, where sudden changes in traffic conditions can be adjusted by a few update steps to obtain a quality approximate solution. We demonstrate how extending the network may result in increased congestion and a degradation in system performance, highlighting the importance of a careful selection of the most beneficial roads to add, which will be the subject of future research. Balancing the traffic flow by influencing user route choices offers a less costly and more flexible solution to the congestion problem. Our experiments on macroscopic traffic-flow optimization by giving instantaneous and localized routing advice demonstrates its potential for improvements in system performance. The framework also allows for the study of balancing demand by scheduling departure times, which could be integrated into our optimization framework; this is one of the future directions for a follow-up study. These extensions can be tested at a low computational cost using our model and optimization method without the need for expensive largescale agent-based simulations. Other possible generalizations include the introduction of a spill-back mechanism, the integration of more nonlocal traffic condition information, and cases of multiple destinations.

Acknowledgments. The map data are copyrighted by OpenStreetMap contributors and is available from [41]. B.L. and D.S. acknowledge support from the Leverhulme Trust (RPG-2018-092), European Union's Horizon 2020 research and innovation programme under the Marie SkłodowskaCurie Grant Agreement No. 835913. D.S. acknowledges support from the EPSRC programme grant TRANSNET (EP/R035342/1). A.Y.L. acknowledges support from the Laboratory Directed Research and Development program of Los Alamos National Laboratory under Projects No. 20190059DR and No. 20200121ER.
[1] D. Schrank, B. Eisele, and T. Lomax, 2019 urban mobility report, https://mobility.tamu.edu/umr/report/ (2019).

[2] J. Gorzelany, The world's most traffic congested cities, Forbes, https://www.forbes.com/sites/jimgorzelany/2013/04/25/theworlds-most-traffic-congested-cities/ (2013).
[3] Department for Transport, Transport statistics Great Britain: 2018, https://assets.publishing.service.gov.uk/government/ uploads/system/uploads/attachment_data/file/795336/tsgb2018.pdf (2018). 
[4] S. Akorede, Employees in Lagos are stressed, burned out and exhausted because of "hellish traffic," CNN, https://edition.cnn.com/travel/article/traffic-stress-lagosnigeria/index.html (2019).

[5] S. Peeta and A. K. Ziliaskopoulos, Foundations of dynamic traffic assignment: The past, the present and the future, Netw. Spat. Econ. 1, 233 (2001).

[6] A. Hamilton, B. Waterson, T. Cherrett, A. Robinson, and I. Snell, The evolution of urban traffic control: Changing policy and technology, Transp. Plan. Technol. 36, 24 (2013).

[7] S. Çolak, A. Lima, and M. C. González, Understanding congested travel in urban areas, Nat. Commun. 7, 10793 (2016).

[8] J. Alonso-Mora, S. Samaranayake, A. Wallar, E. Frazzoli, and D. Rus, On-demand high-capacity ride-sharing via dynamic trip-vehicle assignment, Proc. Natl. Acad. Sci. USA 114, 462 (2017).

[9] S. Lim, H. Balakrishnan, D. Gifford, S. Madden, and D. Rus, Stochastic motion planning and applications to traffic, Int. J. Rob. Res. 30, 699 (2011).

[10] S. Thrun, M. Montemerlo, H. Dahlkamp, D. Stavens, A. Aron, J. Diebel, P. Fong, J. Gale, M. Halpenny, G. Hoffmann et al., Stanley: The robot that won the DARPA grand challenge, J. Field Rob. 23, 661 (2006).

[11] D. J. Fagnant and K. Kockelman, Preparing a nation for autonomous vehicles: Opportunities, barriers and policy recommendations, Transp. Res. Part A: Policy Pract. 77, 167 (2015).

[12] J. Macfarlane, When apps rule the road: The proliferation of navigation apps is causing traffic chaos. It's time to restore order, IEEE Spectrum 56, 22 (2019).

[13] C. Wu, A. Kreidieh, E. Vinitsky, and A. M. Bayen, Emergent behaviors in mixed-autonomy traffic, in Proceedings of the 1st Annual Conference on Robot Learning, edited by S. Levine, V. Vanhoucke, and K. Goldberg, Proceedings of Machine Learning Research Vol. 78 (PMLR, Mountain View, CA, 2017), pp. 398-407.

[14] K. Nagel and M. Schreckenberg, A cellular automaton model for freeway traffic, J. Phys. I France 2, 2221 (1992).

[15] H. Bar-Gera, Origin-based algorithms for transportation network modeling, Ph.D. thesis, University of Illinois at Chicago, 1999.

[16] T. Roughgarden, Selfish Routing and the Price of Anarchy (MIT Press, Cambridge, MA, 2005).

[17] W. Y. Szeto and S. C. Wong, Dynamic traffic assignment: Model classifications and recent advances in travel choice principles, Cent. Eur. J. Eng. 2, 1 (2012).

[18] T. L. Friesz, J. Luque, R. L. Tobin, and B.-W. Wie, Dynamic network traffic assignment considered as a continuous time optimal control problem, Oper. Res. 37, 893 (1989).

[19] E. Ben-Elia and Y. Shiftan, Which road do I take? A learning-based model of route-choice behavior with realtime information, Transp. Res. Part A: Policy Pract. 44, 249 (2010).

[20] E. Ben-Elia, R. Di Pace, G. N. Bifulco, and Y. Shiftan, The impact of travel information's accuracy on route-choice, Transp. Res. Part C: Emerg. Technol. 26, 146 (2013).
[21] M. Ben-Akiva, A. De Palma, and K. Isam, Dynamic network models and driver information systems, Transp. Res. Part A: Gen. 25, 251 (1991).

[22] M. Kuwahara and T. Akamatsu, Decomposition of the reactive dynamic assignments with queues for a many-to-many origindestination pattern, Transp. Res. B: Method. 31, 1 (1997).

[23] A. J. Pel, M. C. J. Bliemer, and S. P. Hoogendoorn, Hybrid route choice modeling in dynamic traffic assignment, Transp. Res. Rec. 2091, 100 (2009).

[24] Electronic road pricing, https://web.archive.org/web/20110605 101108/http://www.lta.gov.sg/motoring_matters/index_ motoring_erp.htm (2019).

[25] N. Barak, Israel tries battling traffic jams with cash handouts, ISRAEL21c, https://www.israel21c.org/israel-triesbattling-traffic-jams-with-cash-handouts/ (2019).

[26] B. D. Greenshields, Studying traffic capacity by new methods, J. Appl. Psychol. 20, 353 (1936).

[27] See Supplemental Material at http://link.aps.org/supplemental/ 10.1103/PhysRevResearch.2.032059 for details, which includes Refs. [39,40].

[28] P.-A. Chen and D. Kempe, Altruism, selfishness, and spite in traffic routing, in Proceedings of the 9th ACM Conference on Electronic Commerce (ACM Press, New York, 2008), pp. 140-149.

[29] G. Zeng, D. Li, S. Guo, L. Gao, Z. Gao, H. E. Stanley, and S. Havlin, Switch between critical percolation modes in city traffic dynamics, Proc. Natl. Acad. Sci. USA 116, 23 (2019).

[30] OpenStreetMap contributors, Planet dump retrieved from https: //planet.osm.org,https://www.openstreetmap.org (2017).

[31] A. Karduni, A. Kermanshah, and S. Derrible, A protocol to convert spatial polyline data to network formats and applications to world urban road networks, Sci. Data 3, 160046 (2016).

[32] D. Braess, Über ein paradoxon aus der verkehrsplanung, Unternehmensforschung 12, 258 (1968).

[33] J. E. Cohen and P. Horowitz, Paradoxical behaviour of mechanical and electrical networks, Nature (London) 352, 699 (1991).

[34] D. Witthaut and M. Timme, Braess's paradox in oscillator networks, desynchronization and power outage, New J. Phys. 14, 083036 (2012).

[35] G. M. Donovan, Biological version of Braess' paradox arising from perturbed homeostasis, Phys. Rev. E 98, 062406 (2018).

[36] F. L. Chernousko and A. A. Lyubushin, Method of successive approximations for solution of optimal control problems, Optim. Control Appl. Methods 3, 101 (1982).

[37] A. Y. Lokhov and D. Saad, Optimal deployment of resources for maximizing impact in spreading processes, Proc. Natl. Acad. Sci. USA 114, E8138 (2017).

[38] Q. Li, L. Chen, C. Tai, and W. E, Maximum principle based algorithms for deep learning, J. Mach. Learn. Res. 18, 1 (2017).

[39] A. Wächter and L. T. Biegler, On the implementation of an interior-point filter line-search algorithm for large-scale nonlinear programming, Math. Program. 106, 25 (2006).

[40] I. Dunning, J. Huchette, and M. Lubin, Jump: A modeling language for mathematical optimization, SIAM Rev. 59, 295 (2017).

[41] https://www.openstreetmap.org. 